\title{
ON A RADIUS PROBLEM CONCERNING A CLASS OF CLOSE-TO-CONVEX FUNCTIONS
}

\author{
RICHARD FOURNIER \\ Département de Mathématiques et de Statistique, Université de Montréal \\ Montréal (QC) HзC 3J\%, Canada
}

\begin{abstract}
The problem of estimating the radius of starlikeness of various classes of closeto-convex functions has attracted a certain number of mathematicians involved in geometric function theory ([7], volume 2, chapter 13). Lewandowski [11] has shown that normalized closeto-convex functions are starlike in the disc $|z|<4 \sqrt{2}-5$. Krzyż [10] gave an example of a function $f(z)=z+\sum_{n=2}^{\infty} a_{n} z^{n}$, non-starlike in the unit disc $\mathbb{D}$, and belonging to the class

$$
H=\left\{f \mid f^{\prime}(\mathbb{D}) \text { lies in the right half-plane. }\right\}
$$

More generally let

$$
H^{*}=\left\{f \mid f^{\prime}(\mathbb{D}) \text { lies in some half-plane not containing } 0 .\right\}
$$

To the best of our knowledge, the radii of starlikeness of both $H$ and $H^{*}$ are still unknown, in spite of the fact that corresponding extremal functions can be described in a relatively simple way (by using, for example, Ruscheweyh's duality theory [15]).

This paper is a survey of recent results concerning the radius of starlikeness of

$$
K=\left\{f \in H|| f^{\prime}(z)-1 \mid<1, z \in \mathbb{D}\right\} .
$$

1. Introduction. Let $A_{0}$ denote the set of functions $f$ analytic in the unit $\operatorname{disc} \mathbb{D}=\{z|| z \mid<1\}$ and normalized by $f(0)=f^{\prime}(0)-1=0$. Let $K$ denote the subset of $A_{0}$ whose members satisfy

$$
\left|f^{\prime}(z)-1\right|<1, \quad z \in \mathbb{D} .
$$

It is easily seen that each function $f \in K$ is univalent; in fact $f(\mathbb{D})$ is a closeto-convex domain of the complex plane. The radius $r_{K}$ of starlikeness of $K$ is

1991 Mathematics Subject Classification: Primary 30C45.

We would like to thank Prof. J. Krzyż for his kind invitation to the Banach Center in the fall of 1992. Most numerical estimations given in this paper were performed by St. Ruscheweyh, we would like to thank him for interesting discussions. Support from an FCAR (Quebec) grant is acknowledged.

The paper is in final form and no version of it will be published elsewhere.
\end{abstract}


defined as the radius of the largest disc centered at the origin whose image by an arbitrary $f \in K$ is a starlike domain with respect to the origin. In other words (we refer to [4] for basic facts concerning univalent functions)

$$
r_{K}=\sup \left\{r \in(0,1) \mid \operatorname{Re}\left(\frac{z f^{\prime}(z)}{f(z)}\right)>0 \text { if }|z|<r \text { and } f \in K\right\} .
$$

The estimate $2 / \sqrt{5} \leq r_{K}$ was first obtained by MacGregor [12] and also appeared later in several papers in the litterature (see in particular [5], [14]). In fact most elementary approaches to the problem of estimating $r_{K}$ lead to $2 / \sqrt{5} \leq r_{K}$. It was found later [5], [6] that the constant $2 / \sqrt{5}$ is sharp with respect to a related problem, namely

(1) $\frac{2}{\sqrt{5}}=\sup \left\{\lambda \in(0,1) \mid f(\Delta)\right.$ is starlike if $f \in A_{0}$ and $\left.\left|f^{\prime}(z)-1\right|<\lambda, z \in \mathbb{D}\right\}$.

It follows in particular from (1) that $r_{K}<1$. Mocanu [14] also exhibited a non-starlike function in $\mathrm{K}$. The best available estimate is $.974<r_{K}$, and this is due to V. Singh [18]. A simple compactness argument shows that there must exist a function $f_{K} \in K$ such that the image of a disk of radius $r$ with center at the origin is starlike if and only if $0<r \leq r_{K}$. Due to the methods used in [5], [12], [14] or [18], the exact nature of $f_{K}$ is not well known. The main goal of this paper is to survey several methods leading to the fact that $f_{K}^{\prime}-1$ is a (finite) Blaschke product. Some numerical experimentations strongly suggest that $f_{K}^{\prime}-1$ is a Blaschke product of order 2 and that $r_{K}$ is amazingly close to one, in fact $.996<r_{K}<1$.

Let us introduce some more definitions. Let $\mathcal{B}$ denote the unit ball (in the supnorm) of $H(\mathbb{D})$ and $\mathcal{B}_{0}$ the set of functions $w \in \mathcal{B}$ vanishing at the origin. Any $f \in K$ admits a representation $f^{\prime}-1=w$ where $w \in \mathcal{B}_{0}$. A simple computation leads to

$$
\begin{aligned}
r_{K}=\sup \left\{r \in(0,1)|| w(r)+\frac{1}{r} \int_{0}^{r} w(t) d t \mid\right. & \\
& \left.+\left|w(r)-\frac{1}{r} \int_{0}^{r} w(t) d t\right| \leq 2, w \in \mathcal{B}_{0}\right\} .
\end{aligned}
$$

We also define for each $0 \leq \rho \leq 1,0<r<1,|\xi|=1$, in accordance with Schwarz lemma,

$$
\begin{aligned}
\mathcal{B}_{0}(r, \rho, \xi) & =\left\{w \in \mathcal{B}_{0} \mid w(r)=r \rho \xi\right\}, \\
I(r, \rho, \xi) & =\left\{\frac{1}{r} \int_{0}^{r} w(t) d t \mid w \in \mathcal{B}_{0}(r, \rho, \xi)\right\} .
\end{aligned}
$$

It follows from (2) that

$$
r_{K}=\sup \left\{r \in(0,1) \mid I(r, \rho, 1) \subseteq \varepsilon_{r, \rho}, \rho \in[0,1]\right\}
$$


where $\varepsilon_{r, \rho}$ represents the interior of the ellipse with equation

$$
|u-r \rho|+|u+r \rho|=2 .
$$

For the sake of completeness we will give yet another proof of the estimate $2 \sqrt{5} \leq r_{K}$. Any function $w \in \mathcal{B}_{0}(r, \rho, \xi)$ admits a representation

$$
w(z)=z \frac{\frac{z-r}{1-r z} W(z)+\rho \xi}{1+\rho \bar{\xi} \frac{z-r}{1-r z} W(z)}, \quad \text { where } \quad W \in \mathcal{B}
$$

and therefore, for each fixed $r \in(0,1)$,

$$
\max _{w \in \mathcal{B}_{0}(r, \rho, \xi)}\left|\frac{1}{r} \int_{0}^{r} w(t) d t\right|=\frac{1}{r} \int_{0}^{r} t \frac{\frac{r-t}{1-t r}+\rho}{1+\rho \frac{r-t}{1-t r}} d t
$$

is an increasing function of $\rho \in(0,1)$ bounded above by $r / 2$. Because the minor semi-axis of the ellipse $\partial \varepsilon_{r, \rho}$ has length $\sqrt{1-r^{2} \rho^{2}}$, we obtain from (3)

$$
r_{K} \geq \sup \left\{r \in(0,1) \mid \frac{r}{2} \leq \sqrt{1-r^{2}}\right\}=\frac{2}{\sqrt{5}} .
$$

This argument shows geometrically why $2 / \sqrt{5}(\sim .89)$ is such a crude lower bound for $r_{K}$. It also shows why a better knowledge of the boundary points of the convex set $I(r, \rho, 1)$ is needed. These boundary points correspond to functions (compare with (4)) maximizing over $\mathcal{B}$ the real part of a functional of the type

$$
L(W)=\int_{0}^{r} t \frac{\frac{t-r}{1-r t} W(t)+\rho \xi}{1+\rho \bar{\xi} \frac{t-r}{1-r t} W(t)} d t
$$

This approach, conbined with results due to Cochrane and MacGregor (2), will be exploited in section 3 , in order to prove that $f_{K}^{\prime}-1$ is a finite Blaschke product.

2. On V. Singh's estimate. The estimate $r_{K}>.974$ has been obtained in [18] as the result of elementary but clever computations. It is based on the following inequality, valid for any $w \in \mathcal{B}_{0}, r \in(0,1)$ and $t \in(0,1)$ :

(6) $\left|w(t r)-w(r) \frac{t\left(1-r^{2}\right)\left(1-r^{2} t^{2}\right)}{\left(1-r^{2} t\right)^{2}-(1-t)^{2}|w(r)|^{2}}\right| \leq \frac{t(1-t)\left(1-t r^{2}\right)\left(r^{2}-|w(r)|^{2}\right)}{\left(1-r^{2} t\right)^{2}-(1-t)^{2}|w(r)|^{2}}$.

Singh obtained this inequality, which is sharp for each admissible value of $t$ and $r$, by using the Schwarz lemma. It follows from (6), for each $r \in(0,1), \rho \in[0,1]$ and $\xi \in \partial \mathbb{D}$, that $I(r, \rho, \xi)$ is contained in a closed disc $D(r, \rho, \xi)$ with center $r \rho \xi\left(1-r^{2}\right) \int_{0}^{1} \frac{t\left(1-r^{2} t^{2}\right)}{\left(1-r^{2} t\right)^{2}-(1-t)^{2} r^{2} \rho^{2}} d t$ and radius $r^{2}\left(1-\rho^{2}\right) \int_{0}^{1} \frac{t(1-t)\left(1-t r^{2}\right)}{\left(1-r^{2} t\right)^{2}-(1-t)^{2} r^{2} \rho^{2}} d t$. As a matter of fact Singh even claims that $I(r, \rho, \xi)=D(r, \rho, \xi)$ for each admissible value of $r, \rho$ and $\xi$. This is easily seen to be true for example when $\rho=0$ or 1 , and in principle it should be enough in order to compute $r_{K}$ precisely. However Singh's claim cannot be true in general, and we would like to explain why. By (6) and the triangle inequality for integrals the claim amounts to the fact that, for 
any $r, \rho \in(0,1)$ and $\psi \in[0,2 \pi]$, there must exist a function $\varphi \in \mathcal{B}_{0}(r, \rho, 1)$ such that

$$
\varphi(t r)=\frac{\varphi(r) \frac{t r}{r}\left(1-r^{2}\right)\left(1-(t r)^{2}\right)+e^{i \psi} \frac{t r}{r}(1-r(t r))\left(r^{2}-|\varphi(r)|^{2}\right)\left(1-\frac{t r}{r}\right)}{(1-r(t r))^{2}-\left(1-\frac{t r}{r}\right)^{2}|\varphi(r)|^{2}}
$$

holds for all $t \in[0,1]$. Because $\varphi$ is analytic, we must have

$$
\frac{\varphi(z)}{z}=\frac{\rho\left(1-r^{2}\right) \frac{1-z^{2}}{(1-r z)^{2}}-\left(1-\rho^{2}\right) \frac{z-r}{1-r z} e^{i \psi}}{1-\left(\frac{z-r}{1-r z}\right)^{2} \rho^{2}}
$$

for all $z \in \mathbb{D}$. By a passage to $|z|=1$, we obtain

$$
\frac{\varphi\left(\frac{e^{i \theta}+r}{1+r e^{i \theta}}\right)}{\frac{e^{i \theta}+r}{1+r e^{i \theta}}}=\frac{\rho\left(1-e^{i \theta}\right)-\left(1-\rho^{2}\right) e^{i(\theta+\varphi)}}{1-e^{2 i \theta} \rho^{2}}
$$

for almost all $\theta \in[0,2 \pi]$. If in particular we choose $\rho=\sqrt{2}-1, \theta=\pi / 4$ and $\psi=\pi / 2$ we obtain

$$
\left|\varphi\left(\frac{e^{i \theta}+r}{1+r e^{i \theta}}\right)\right|=\left|\frac{1-i}{1-i \rho^{2}}\right|>1
$$

which is of course impossible.

In spite of the fact that (6) does not seem strong enough to lead to an exact characterization of the regions $I(r, \rho, \xi)$, it is nevertheless strong enough to obtain the estimate

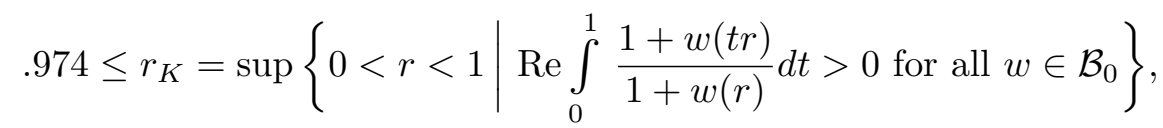

which is a serious improvement upon $r_{K} \geq 2 / \sqrt{5}$. Moreover there is numerical evidence that Singh's method leads to sharp estimates for

$$
\inf _{w \in \mathcal{B}_{0}} \operatorname{Re} \int_{0}^{1} \frac{1+w(t r)}{1+w(r)} d t
$$

for fixed $r<.975$. This is rather surprising, and for now we can't explain it.

3. $f_{K}^{\prime}-1$ is a finite Blaschke product. In this section we show how some results due to Cochrane and MacGregor [2] already imply that $f_{K}^{\prime}-1$ is a finite Blaschke product. Assume $L$ is a complex valued continuous functional on $\mathcal{B}$. Then $L$ is called Fréchet differentiable at $W_{0} \in \mathcal{B}$ relative to $\mathcal{B}$ if, for any variation $W^{*}=W_{0}+\varepsilon \tilde{W}+o(\varepsilon) \in \mathcal{B}$,

$$
L\left(W^{*}\right)=L\left(W_{0}\right)+\varepsilon L_{W_{0}}(\tilde{W})+o(\varepsilon)
$$

where $L_{W_{0}}$ is a continuous linear functional (called the Fréchet derivative) defined over the set $H(\mathbb{D})$ of all analytic functions on $\mathbb{D}$. In the definition of the variation $W^{*}, o(\varepsilon)$ represents a function of $z$ and $\varepsilon$, such that $\lim _{\varepsilon \rightarrow 0} o(\varepsilon) / \varepsilon=o$ uniformly as long as $z$ is restricted to a compact subset of $\mathbb{D}$. In $(8) o(\varepsilon)$ is a quantity such 
that $\lim _{\varepsilon \rightarrow 0} o(\varepsilon) / \varepsilon=o$. Cochrane and MacGregor [2](see also [9]) proved the following

Theorem 1. Let $L$ be a continuous functional on $\mathcal{B}$, and $W_{0} \in \mathcal{B}$ such that

$$
\operatorname{Re} L\left(W_{0}\right)=\max _{W \in \mathcal{B}} \operatorname{Re} L(W) .
$$

Assume that $L$ has a Fréchet derivative at $W_{0}$ relative to $\mathcal{B}$, and that this derivative does not vanish identically over $H(\mathbb{D})$. Then $W_{0}$ is a finite Blaschke product.

This result is important to us because the functional $L$ defined in (5) admits, at any $W_{0} \in \mathcal{B}$, the Fréchet derivative

$$
L_{W_{0}}(h)=\int_{0}^{r} \frac{\left(1-\rho^{2}\right) \frac{t-r}{1-r t} t h(t)}{\left(1+\rho \bar{\xi} \frac{t-r}{1-r t} W_{0}(t)\right)^{2}} d t
$$

which is not (except when $\rho=1$ ) the zero functional over $H(\mathbb{D})$. It therefore follows that any function maximizing $\operatorname{Re} L$ is a finite Blaschke product (and this holds trivially even if $\rho=1$ ). It should now be clear from the discussion in our introduction that $f_{K}^{\prime}-1$ is a finite Blaschke product. It seems however quite difficult to use the Cochrane-MacGregor method as to obtain any bound on the order of the Blaschke product $f_{K}^{\prime}-1$ (as a matter of fact their method can be used, for certain types of functionals only, to obtain upper bounds on the order of the involved Blaschke products, see [2], Theorems 1 and 2). We shall come back to this topic in our last section.

4. $f_{K}^{\prime}-1$ is a Blaschke product, another proof. In this section we present still another proof of the fact that the real part of the functional $L$ in (5) is maximized over $\mathcal{B}$ by a Blaschke product. Of course this is slightly weaker than the result obtained in section 3 . On the other hand our proof is self-contained and shows a rather surprising connection between our problem and entire functions of exponential type (we refer to [1] for appropriate definitions). We may clearly assume that there exists a function $W_{0} \in \mathcal{B}$, not vanishing identically on $\mathbb{D}$, such that (9) holds.

Let $\varphi \in \mathcal{B}$ such that $\left|W_{0}(z)\right| \leq|\varphi(z)|, z \in \mathbb{D}$. For each real $\psi$, the function $\varphi\left(e^{i \psi} z\right) \frac{W_{0}(z)}{\varphi(z)}$ belongs to $\mathcal{B}$, and the differentiable mapping

$$
\psi \rightarrow \operatorname{Re} L\left(\varphi\left(e^{i \psi} z\right) \frac{W_{0}(z)}{\varphi(z)}\right)
$$

admits a local maximum at $\psi=0$. After simple computations we obtain that

$$
\int_{0}^{r} \frac{t \frac{t-r}{1-r t} W_{0}(t)}{\left(1+\rho \bar{\xi} \frac{t-r}{1-r t} W_{0}(t)\right)^{2}} t \frac{\varphi^{\prime}(t)}{\varphi(t)} d t \quad \text { is real }
$$

for each admissible $\phi$. In a similar manner and for each fixed real $\theta$ the mapping

$$
x \rightarrow \operatorname{Re} L\left(\frac{\varphi(z)+x e^{i \theta}}{1+x e^{-i \theta} \varphi(z)} \frac{W_{0}(z)}{\varphi(z)}\right)
$$


admits a local maximum at $x=0$, when $x$ is restricted to a small interval around the origin. Because $\theta$ is arbitrary we obtain

$$
\int_{0}^{r} \frac{t \frac{t-r}{1-r t} \frac{W_{0}(t)}{\varphi(t)}}{\left(1+\rho \bar{\xi} \frac{t-r}{1-r t} W_{0}(t)\right)^{2}} d t=\int_{0}^{r} \frac{t \frac{t-r}{1-r t} W_{0}(t) \varphi(t)}{\left(1+\rho \bar{\xi} \frac{t-r}{1-r t} W_{0}(t)\right)^{2}} d t
$$

whenever $\varphi$ dominates $W_{0}$.

We now use a factorization $W_{0}(z)=B(z) e^{-F(z)}$ where $B$ is a Blaschke product and $F$ has positive real part over $\mathbb{D}$ with $F(0) \geq 0$. By Herglotz formula

$$
F(z)=F(0) \int_{0}^{2 \pi} \frac{1+z e^{i \theta}}{1-z e^{i \theta}} d \mu(\theta)
$$

for some increasing function $\mu$ over $[0,2 \pi]$ with $\mu(0)=\mu\left(0^{+}\right)=0$ and $\mu(2 \pi)=1$. We assume $F(0)>0$. For each continuous function $c(\theta)$ satisfying $0 \leq c(\theta) \leq$ $F(0)$ over $[0,2 \pi]$, let

$$
F_{c}(z)=\int_{0}^{2 \pi} \frac{1+z e^{i \theta}}{1-z e^{i \theta}} c(\theta) d \mu(\theta) .
$$

Clearly $\left|W_{0}(z)\right| \leq\left|e^{-F_{c}(z)}\right|$ for $z \in \mathbb{D}$ and by (10)

$$
\int_{0}^{2 \pi}\left(\operatorname{Im} \int_{0}^{r} \frac{t \frac{t-r}{1-r t} W_{0}(t)}{\left(1+p \bar{\xi} \frac{t-r}{1-r t} W_{0}(t)\right)^{2}} \frac{t e^{i \theta}}{\left(1-t e^{i \theta}\right)^{2}} d t\right) c(\theta) d \mu(\theta)=0 .
$$

Remark that (12) holds for all monomials $c(\theta)=F(0)\left(\frac{\theta}{2 \pi}\right)^{n}$, and therefore it also holds for an arbitrary real-valued continuous function over $[0,2 \pi]$. We define $U(z)=\int_{0}^{r} \frac{t \frac{t-r}{1-r t} W_{0}(t)}{\left(1+\rho \bar{\xi} \frac{t-r}{1-r t} W_{0}(t)\right)^{2}} \frac{t z}{(1-t z)^{2}} d t$; this function is analytic in the closed unit disc, $U(0)=0$, and $U$ is non-constant since $W_{0} \not \equiv 0$. If $\tilde{\mu}\left(\theta=\int_{0}^{\theta} \operatorname{Im}\left(U\left(e^{i t}\right)\right) d \mu(t)\right.$, then $\tilde{\mu}$ has bounded variation over $[0,2 \pi]$ and by $(12), \int_{0}^{2 \pi} c(\theta) d \tilde{\mu}(\theta)=0$ for any continuous function $c(\theta)$ over $[0,2 \pi]$. Therefore $([13]$, page 230$) \tilde{\mu}$ is constant, $\tilde{\mu}(0)=0$, and we obtain

$$
\int_{\theta_{1}}^{\theta_{2}} \operatorname{Im}\left(U\left(e^{i t}\right)\right) d \mu(t)=0 \quad \text { if } \quad 0 \leq \theta_{1} \leq \theta_{2} \leq 2 \pi .
$$

It can be seen from (13) that $\mu$ is a step-function, and that $\operatorname{Im}\left(U\left(e^{i t}\right)\right)$ vanishes at each point of discontinuity of $\mu$. Because $U$ is non-constant and analytic in $\overline{\mathbb{D}}$, there can only be a finite number of such discontinuities. In other words, we may assume

$$
F(z)=F(0) \sum_{j=1}^{n} \lambda_{j} \frac{1+z e^{i \theta j}}{1-z e^{i \theta j}}
$$

where $F(0)>0,0<\lambda_{j}$ and $\sum_{j=1}^{n} \lambda_{j}=1$. 
For each $x \in(0, F(0))$, let

$$
F_{x}(z)=\lambda_{1} x \frac{1+z e^{i \theta_{1}}}{1-z e^{i \theta_{1}}}
$$

Let also $E$ be defined by

$$
E(z)=\int_{0}^{r} \frac{t \frac{t-r}{1-r t} W_{0}(t)}{\left(1+\rho \bar{\xi} \frac{t-r}{1-r t} W_{0}(t)\right)^{2}} e^{\frac{1+t e^{i \theta_{1}}}{1-t e^{i \theta_{1}} z}} d t .
$$

$E$ is an entire function of exponential type. An application of (10) with $\varphi(z)=$ $e^{-F_{x}(z)}$ shows that, $x$ being arbitrary in $(0, F(0))$,

$$
E(z)=\bar{E}(-z), \quad \text { for all } z \in\left[0, F(0) \lambda_{1}\right] .
$$

Therefore $E(z)$ coïncides with $\bar{E}(-z)$ for all real values of $z$, and for $z<0$

$$
|E(z)| \leq r \max _{t \in[0, r]}\left|\frac{t \frac{t-r}{1-r t} W_{0}(t)}{\left(1+\rho \bar{\xi} \frac{t-r}{1-r t} W_{0}(t)\right)^{2}}\right| e^{-\frac{1-r}{1-r}|z|} .
$$

The last estimate is also valid for $z>0$. In other words $E(z)$ tends to zero exponentially as $z$ tends to infinity along the real axis. By a known result ([1], page 69 ) we have $E(z) \equiv 0$. Because $W_{0}$ does not vanish identically, we must conclude that $F(0)=0$, and $W_{0}$ is a finite Blaschke product. Blaschke products being extreme points of $\mathcal{B}$ (see [3]), we may conclude that the functional $L$ from (5) has its real part maximized over $\mathcal{B}$ by a unique Blaschke product.

5. A conjecture of St. Ruscheweyh. As indicated in section 3, there does not seem to exist a general theory of extremal problems capable of predicting what may be the order of the finite Blaschke product $f_{K}^{\prime}-1$. We shall now use an idea due to St. Ruscheweyh (private communication) to deal with this problem.

Let $\mathcal{H}$ denote the set of analytic functions $F(z)$, satisfying $F(0)=1$, with real part greater than $1 / 2$ over the disc $\mathbb{D}$. By Herglotz formula, $\mathcal{H}$ can be identified with the set of probability measures $\mu$ over $[0,2 \pi]$ via the representation

$$
F(z)=\int_{0}^{2 \pi} \frac{1}{1-z e^{i \theta}} d \mu(\theta), \quad z \in \mathbb{D} .
$$

For a given fixed $z \in \mathbb{D} \backslash\{0\}$, let us define $\mathcal{H}_{z}$ as the set of all mappings of the type

$$
h(\xi)=\frac{F(z)}{F(\xi z)}, \quad \xi \in \mathbb{D}, F \in \mathcal{H} .
$$

We now consider a linear functional $L$ defined over $H(\mathbb{D})$. Ruscheweyh's conjecture asserts that $\operatorname{Re} L$ can be maximized over the set $\mathcal{H}_{z}$ by functions $h$ of the type

$$
h(\xi)=\frac{\frac{\lambda}{1-e^{i \theta_{1} z}}+\frac{1-\lambda}{1-e^{i \theta_{2} z}}}{\frac{\lambda}{1-e^{i \theta_{1} \xi z}}+\frac{1-\lambda}{1-e^{i \theta_{2}} \xi z}}
$$


where each $\theta_{j}$ is real and $\lambda \in[0,1]$. Here we assume that $\operatorname{Re} L$ is not constant over $\mathcal{H}_{z}$. The truth of this conjecture implies that $f_{K}^{\prime}-1$ can be thought of as Blaschke product of order 2; this can be understood most easily from the equivalent definition of $r_{K}$ given in (7) and the fact that functions $F$ in $\mathcal{H}$, whose representation (14) is given by a two-step function $\mu$, correspond to Blaschke products of order 2 under the mapping

$$
w \rightarrow \frac{1}{1-w}
$$

which transforms $\mathcal{B}_{0}$ onto $\mathcal{H}$.

Numerical computations suggest that the conjecture may be true. The conjecture is also reminiscent of a result due to Ruscheweyh [16] concerning the variability region of a quotient of linear functionals over the class $\mathcal{H}$. We end this paper by proving a weaker form of the conjecture. Let $L$ and $z$ as above, and $F_{0} \in \mathcal{H}$ such that

$$
\max _{F \in \mathcal{H}} \operatorname{Re} L\left(\frac{F(z)}{F(\xi z)}\right)=\operatorname{Re} L\left(\frac{F_{0}(z)}{F_{0}(\xi z)}\right) .
$$

We shall prove that

$$
F_{0}(\xi)=\sum_{j=0}^{n} \frac{\lambda_{j}}{1-e^{i \theta_{j} \xi}}, \quad \theta_{j} \text { real, } n \geq 1,0<\lambda_{j}<1 \text { and } \sum_{j=1}^{n} \lambda_{j}=1 .
$$

This means again of course that $f_{K}^{\prime}-1$ is a finite Blaschke product!

According to $(15)$ and the convexity of $\mathcal{H}$, we obtain for any $t \in(0,1)$ and $F \in \mathcal{H}$

$$
\begin{aligned}
& \operatorname{Re} L\left(\frac{(1-t) F_{0}(z)+t F(z)}{(1-t) F_{0}(\xi z)+t F(\xi z)}\right) \\
& \quad=\operatorname{Re} L\left(\frac{F_{0}(z)}{F_{0}(\xi z)}\right)+t \operatorname{Re} L\left(\frac{F(z) F_{0}(\xi z)-F(\xi z) F(\xi z) F_{0}(z)}{F_{0}(\xi z)^{2}}\right)+o(t) \\
& \quad \leq \operatorname{Re} L\left(\frac{F_{0}(z)}{F_{0}(\xi z)}\right) .
\end{aligned}
$$

We define a continuous linear functional $L^{*}$ over $H(\mathbb{D})$ by

$$
L^{*}(F)=L\left(\frac{F(z) F_{0}(\xi z)-F(\xi z) F_{0}(z)}{F_{0}(\xi z)^{2}}\right) .
$$

By (17), $\operatorname{Re} L^{*}(F) \leq 0=\operatorname{Re} L^{*}\left(F_{0}\right)$. It can also be checked that $\operatorname{Re} L^{*}$ is not constant over $\mathcal{H}$ because $\operatorname{Re} L$ is not constant over $\mathcal{H}_{z}$. It therefore follows from a Theorem of Hallenbeck and MacGregor [8] concerning the so-called support points of $\mathcal{H}$ that $F_{0}$ must be of the form prescribed by (16). Finally, note that another proof of this fact can be obtained by using directly the measures $\mu$ in (14) and the Toeplitz representation [17] of linear functionals over $H(\mathbb{D})$. 


\section{References}

[1] R. P. Boas, Entire Functions, Academic Press, New York, 1954.

[2] P. C. Cochrane and T. H. MacGregor, Fréchet differentiable functionals and support points for families of analytic functions, Trans. Amer. Math. Soc. 236 (1978), 75-92.

[3] K. de Leeuw and W. Rudin, Extreme points and extremum problems in $H_{1}$, Pacific J. Math. 8 (1958), 467-485.

[4] P. L. Duren, Univalent Functions, Springer, New York, 1983.

[5] R. Fournier, On integrals of bounded analytic functions in the unit disc, Complex Variables 11 (1989), 125-133.

[6] - The range of a continuous linear functional over a class of functions defined by subordination, Glasgow Math. J. 32 (1990), 381-387.

[7] A. W. Goodman, Univalent Functions, Mariner Publishing Company, Tampa, 1983.

[8] D. J. Hallenbeck and T. H. MacGregor, Support points of families of analytic functions defined by subordination, Trans. Amer. Math. Soc. 278 (1983), 523-546.

[9] _- - Linear Problems and Convexity Techniques in Geometric Function Theory, Pitman, Boston, 1984.

[10] J. Krzyż, A counterexample concerning univalent functions, Folia Soc. Scient. Lubliniensis 2 (1962), 57-58.

[11] Z. Lewandowski, Sur l'identité de certaines classes de fonctions univalentes, Ann. Univ. M. Curie-Skłodowska 14 (1960), 19-46.

[12] T. H. MacGregor, A class of univalent functions, Proc. Amer. Math. Soc. 15 (1964), 311-317.

[13] R. M. McLeod, The Generalized Riemann Integral, Mathematical Association of America, 1980.

[14] P. T. Mocanu, Some starlikeness conditions for analytic functions, Rev. Roumaine Math. Pures Appl. 33 (1988), 117-124.

[15] St. Ruscheweyh, Convolutions in Geometric Function Theory, Les Presses de l'Université de Montréal, Montréal, 1982.

[16] —, Duality for Hadamard products with applications to extremal problems for functions regular in the unit disc, Trans. Amer. Math. Soc. 210 (1975), 63-74.

[17] O. Toeplitz, Die linearen volkommenen Räume der Funktionentheorie, Comment. Math. Helv. 23 (1949), 222-242.

[18] V. Singh, Univalent functions with bounded derivative in the unit disc, Indian J. Pure Appl. Math. 5 (1974), 733-754. 